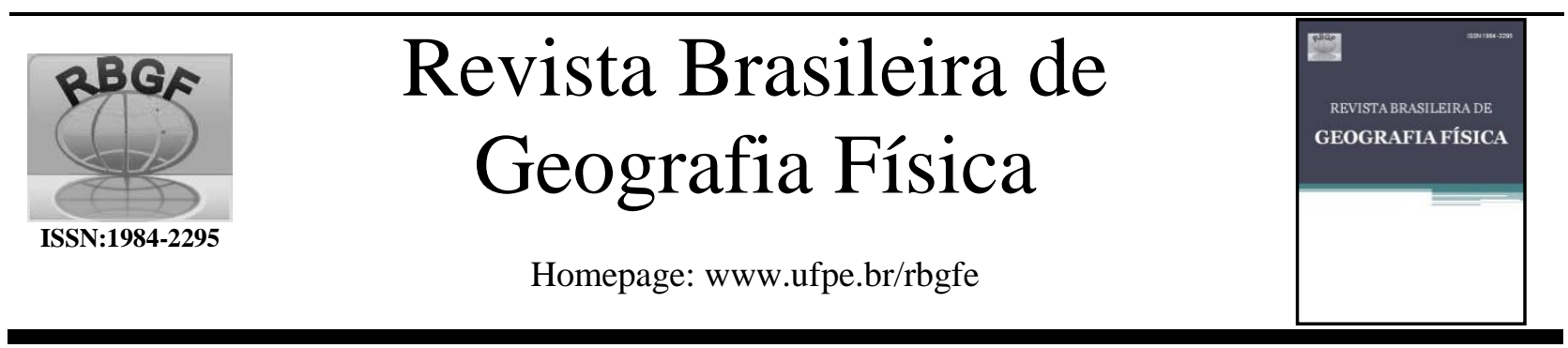

\title{
Modelagem da resposta do escoamento em uma bacia do semiárido da Paraíba utilizando o modelo SWAT
}

\author{
Jennifer Marinho do Nascimento, Tatiane Gomes Frade, Richarde Marques da Silva \\ Departamento de Engenharia Civil e Ambiental/Centro de Tecnologia/UFPB \\ e-mail: jennifer.marinho@hotmail.com
}

Artigo recebido em 27/10/2017 e aceite em 20/06/2018

\begin{abstract}
R E S U M O
Este trabalho teve como objetivo analisar o escoamento superficial na bacia do Alto Rio do Peixe, situada no semiárido do Estado da Paraíba, Brasil. Neste estudo foi utilizado o modelo Soil and Water Assessment Tool (SWAT) para realizar a simulação hidrológica, para isso, foram utilizados dados diários de temperatura, umidade, velocidade do vento da estação meteorológica de São Gonçalo e precipitação no período de 2002-2014, além de mapas de uso e ocupação do solo, tipos de solos e modelo digital de elevação. O período de calibração analisado foi de 2002 a 2010 e a validação de 2011 a 2014, usando dados mensais de vazão da estação fluviométrica de Antenor Navarro. Os resultados obtidos mostraram que as vazões observadas e calculadas foram de $4,7 \mathrm{~m}^{3} / \mathrm{s}$ e $5,8 \mathrm{~m}^{3} / \mathrm{s}$, respectivamente. Os índices de desempenho da calibração foram considerados muito bons $\left(\mathrm{R}^{2}=0,82\right.$ e NS $\left.=0,81\right)$ demonstrando um bom ajuste entre os dados observados e os estimados, enquanto para a validação os resultados de $\mathrm{R}^{2}$ e NS foram de 0,23 e 0,70 , respectivamente.

Palavras-chave: modelagem hidrológica, modelo SWAT, semiárido.
\end{abstract}

\section{Modelling of runoff response in semi-arid catchment of Paraíba State using SWAT}

\begin{abstract}
A B S T R A C T
The objective of this work was to analyze the surface runoff in the Upper Peixe river basin, located in semi-arid of Paraíba State, Brazil. In this study, the SWAT - Soil and Water Assessment Tool was used to perform the hydrological simulation. For this purpose, daily data of temperature, humidity and wind speed of the São Gonçalo meteorological station and precipitation data from 2002-2014 were used. In addition, were used maps of land use and occupation, soil types and digital elevation model. The calibration period was from 2002 to 2010 and the validation period from 2011 to 2014 , using monthly flow data from the Antenor Navarro fluviometric station. The results showed that the observed and calculated flows were $4.7 \mathrm{~m}^{3} / \mathrm{s}$ and $5.8 \mathrm{~m}^{3} / \mathrm{s}$, respectively. The calibration performance values were considered very good $\left(\mathrm{R}^{2}=0.82\right.$ and NS $=0.81$ ), showing a good fit between the observed and estimated data, while for the validation the results of $\mathrm{R}^{2}$ and NS were 0,23 and 0.70 , respectively.
\end{abstract}

Keywords: Hydrological modeling, SWAT model, semiarid.

\section{Introdução}

O aumento do consumo de água no mundo, devido ao crescimento populacional, aumento das atividades produtivas, degradação ambiental dos corpos hídricos e à redução dos totais pluviométricos têm contribuído para a criação de um panorama de escassez hídrica em diversas regiões do planeta (Santos et al., 2017; Marengo, 2006). Segundo a Organização das Nações Unidas até meados de 2030 quase metade da população estará vivendo em uma situação de escassez hídrica
(ONU, 2015), ou seja, os recursos hídricos existentes não conseguirão atender à demanda da população.

Esse problema é ainda mais alarmante para a população residente em zonas áridas $\mathrm{e}$ semiáridas, como é o caso de grande parte do Nordeste do Brasil, e mais especificamente os Estados do Ceará, Paraíba, Pernambuco, Piauí e Rio Grande do Norte, que sofrem com secas cíclicas, que afetam a quantidade de água disponível nos reservatórios da região (Silva et al., 2017). 
O fenômeno natural cíclico da seca assola o Brasil há vários séculos, de modo que a escassez de água no Semiárido não representa qualquer novidade. Considera-se, inclusive, que não é a falta de chuva ou a sua dádiva espacialmente e quantitativamente irregular pela natureza a responsável pelo agravamento das consequências desse fenômeno que vem afetando as condições de vida do povo nordestino, mas é a falta do gerenciamento eficaz dos recursos hídricos desta região que tem sido indicada como a causa principal deste flagelo (Silva, 2003).

Segundo Araújo (2011), a região semiárida brasileira possui clima caracterizado com elevada amplitude térmica com mínima de $15^{\circ} \mathrm{C}$ e máxima de $40^{\circ} \mathrm{C}$, onde a média anual está acima dos $26^{\circ} \mathrm{C}$. A precipitação anual varia entre 280 e $800 \mathrm{~mm} / \mathrm{ano}$. A chuva nessa região é caracterizada por sua concentração em apenas 3 ou 4 meses do ano, podendo ocorrer chuvas de grande intensidade. Nesse período, pode ocorrer até $70 \%$ de toda a precipitação anual, logo os outros 8 ou 9 meses são caracterizados por seca, com precipitações mensais médias de 10 a $15 \mathrm{~mm}$. O comportamento das chuvas possui variações de acordo com o relevo, as condições atmosféricas, exposição aos ventos, os solos e a geologia (Araújo, 2011). As secas que flagelam a região sempre moldaram o comportamento das populações e foram preponderantes para a formulação de políticas públicas regionais.

Do ponto de vista morfoestrutural, o semiárido possui um quadro natural que limita suas potencialidades de disponibilidade hídrica para a população. Caracteriza-se pela aridez do clima, deficiência hídrica, irregularidade nas precipitações, solos com risco de degradação ambiental e limitações agropecuárias (Lima et al., 2011).

A vegetação predominante é a do bioma caatinga, com estratos herbáceos, arbustivos e arbóreos. Como as taxas de evaporação e evapotranspiração predominam sobre as de precipitação, as plantas da região permanecem em estado de latência até começar a quadra chuvosa. Os solos da região são predominantemente secos e pedregosos, sendo, portanto, pouco desenvolvidos (Dantas et al., 2015). Segundo Cirilo (2008), no que se refere à ocorrência de águas subterrâneas, como o território nordestino é em mais de $80 \%$ constituído por rochas cristalinas, há predominância de águas com teor elevado de sais captado em poços de baixa vazão: da ordem de 1 $\mathrm{m}^{3} / \mathrm{h}$. Exceção ocorre nas formações sedimentares, onde as águas normalmente são de melhor qualidade e podem-se extrair maiores vazões, da ordem de dezenas a centenas de $\mathrm{m}^{3} / \mathrm{h}$, de forma contínua.

Diante do exposto, a escassez hídrica é uma realidade no semiárido, o que torna necessário a realização de estudos de modelagem hidrológica para se conhecer o funcionamento do balanço hídrico e o comportamento hidrológico mediante modelos hidrológicos. Modelos hidrológicos que são ferramenta produzidas para avaliar os processos hidrológicos nas escalas espaciais e temporais (Andrade et al., 2013), facilitado pelo acoplamento com Sistemas de Informações Geográficas (Rennó e Soares, 2008), como é o caso do modelo Soil and Water Assessment Tool (SWAT) (Arnold e Allen, 1996). Esse modelo tem sido adaptado, desde o seu surgimento, para algumas áreas específicas e foi integrado ao SIG possibilitando a entrada do banco de dados, a elaboração e edição de cenários ambientais e sua representação em forma de gráficos e mapas, controle e simulações, além de extrair e organizar as saídas do modelo (Bressiani et al., 2015b).

Há alguns anos, o modelo SWAT vem sendo amplamente utilizado em estudos de recursos hídricos e equivalentes (Bressiani et al., 2015a). Podem ser citadas pesquisas em análises hidrológicas em bacias hidrográficas, análise de erosão hídrica em regiões semiáridas, análise da estabilidade lateral em ambientes fluviais, entre outras. Essas pesquisas visam o desenvolvimento da utilização, distribuição e planejamento dos recursos hídricos, bem como da própria região em si. As regiões em situações críticas são áreas de estudo bastante utilizadas, pois busca-se uma melhoria nas condições socioeconômicas das mesmas.

Face ao exposto, este estudo teve como objetivo testar o modelo hidrológico SWAT, seguindo uma sequência de procedimentos recomendada (análise de sensibilidade, calibração e validação), na bacia do Alto Rio do Peixe, a qual é representativa das condições geológicas e climáticas de uma bacia do semiárido do Nordeste do Brasil.

\section{Material e métodos}

\section{Área de estudo}

A área estudada foi a bacia do Alto Rio do Peixe, localizada entre as latitudes $6^{\circ} 20^{\prime} 47^{\prime \prime} \mathrm{S}$ e $7^{\circ} 03^{\prime} 53^{\prime \prime} \mathrm{S}$ e longitudes $37^{\circ} 57^{\prime} 52^{\prime \prime} \mathrm{W}$ e $38^{\circ} 46^{\prime} 48^{\prime \prime}$ $\mathrm{W}$ (Figura 1), inserida mais precisamente no extremo noroeste do Estado da Paraíba, na mesorregião do Sertão, sendo uma das sub-bacias do rio Piranhas-Açu. A bacia do Alto Rio do Peixe 
é uma sub-bacia do rio Piranhas e representa uma das áreas mais promissoras para a expansão agropecuária do Estado da Paraíba (Brandão, 2005). Observa-se também nesta bacia uma grande vocação para as atividades industriais, com o beneficiamento e agregação de valores aos seus

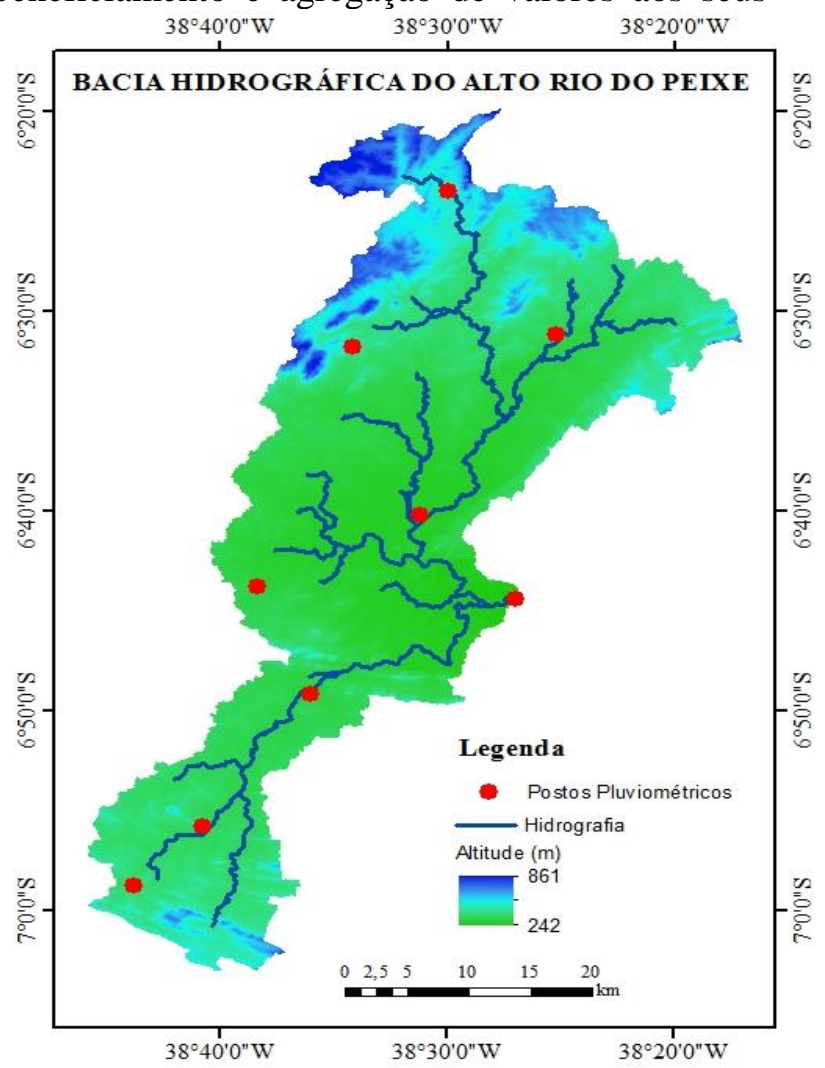

produtos agropecuárias. A bacia tem importância estratégica para o abastecimento alimentar e oferta de serviços, não apenas para os municípios que a compõem, mas também para os estados vizinhos do Ceará e Rio Grande do Norte (Pinto, 1998).

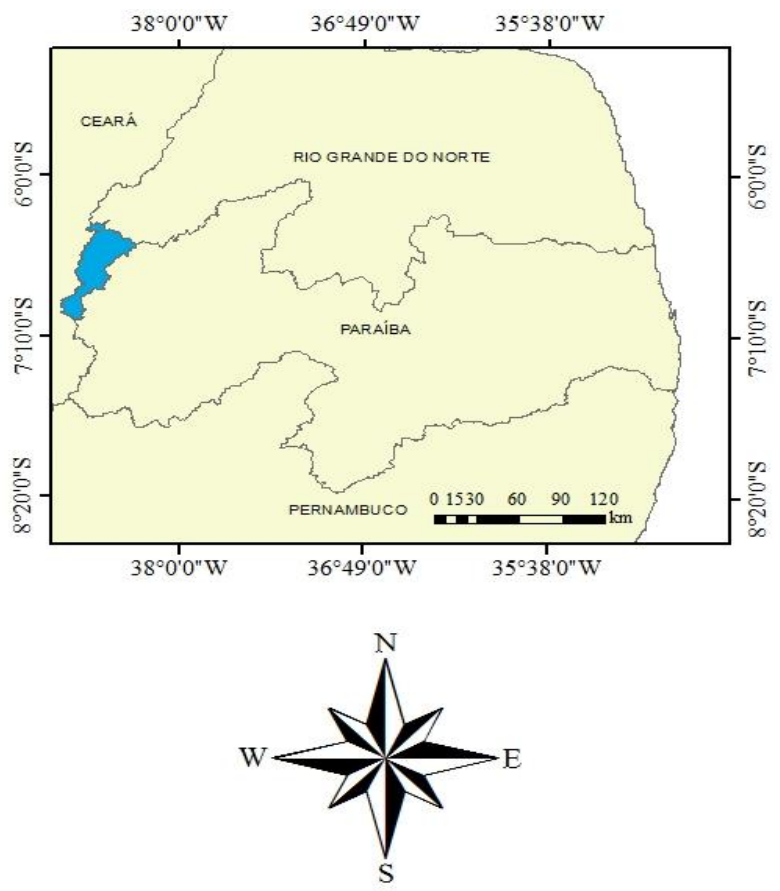

SISTEMA DE PROJEÇÃO LAT./LONG DATUM SIRGAS 2000

Figura 1. Localização da bacia do Alto Rio do Peixe e dos postos pluviométricos utilizados neste estudo. Fonte: IBGE (2015).

No que tange as características climáticas, a classificação de Köppen para a bacia do Alto Rio do Peixe é do tipo Tropical quente e úmido com chuvas de verão-outono ( $A w '$ '), além de ser caracterizado por possuir duas estações (uma seca e outra chuvosa). No que se refere a vegetação, essa bacia apresenta uma predominância do tipo caatinga hiper-xerófila (Brandão, 2005). Quanto aos tipos de solo na bacia, de acordo com EMBRAPA (2006) são encontrados quatro tipos de solo na bacia do Alto Rio do Peixe, sã eles: Luvissolo, Neossolo litólico, Planossolo e Vertissolo (Quadro 1). Esses tipos de solo apresentam grande diversidade: mais rasos $\mathrm{e}$ pedregosos nas áreas mais elevadas e declivosas (Luvissolo e Neossolo Litólico); medianamente profundos, pedregosos, argilosos (Luvissolo) e arenosos (Planossolo), em relevo suave ondulado e muito argiloso e plano (Vertissolo) na bacia sedimentar cretácia, ao longo da rede principal da drenagem (BRASIL, 1972).

\section{Dados hidro-climatológicos}

Para o estudo foram utilizados dados diários para o período de 2002 a 2015 de nove postos pluviométricos e um fluviométrico, além de uma estação meteorológica (Quadro 2). Os dados climatológicos diários usados foram: (a) temperatura máxima, (b) temperatura mínima,

Cobertura do solo, tipos de solo e modelo de elevação da bacia

As Figuras $2 \mathrm{a}-\mathrm{c}$ mostram os mapas de uso e cobertura do solo, tipos de solos e declividade da área de estudo. O mapa de uso e cobertura do solo foi elaborado mediante um mosaico de imagens do satélite Landsat-5 (sensor TM, órbita 215, ponto 65, de 18/06/1990), obtida junto ao Instituto Nacional de Pesquisas Espaciais (INPE). Para esse mapeamento foi utilizada a classificação supervisionado usando o método Máxima Verossimilhança. Foram determinadas cinco classes com base nas interpretações das imagens e verificadas em visitas de campo: (a) corpo hídrico, 
Revista Brasileira de Geografia Física v11, n.03 (2018) 1137-1150.

(b) vegetação arbustiva, (c) vegetação herbácea, (d) vegetação arbórea/arbustiva e (e) solo exposto.

Quadro 1. Tipos de solo para a bacia do Alto Rio do Peixe

\begin{tabular}{|c|c|c|l|}
\hline $\begin{array}{c}\text { Tipos de } \\
\text { solo }\end{array}$ & $\begin{array}{c}\text { Área } \\
\left(\mathrm{km}^{2}\right)\end{array}$ & $\begin{array}{c}\text { Área } \\
(\%)\end{array}$ & \multicolumn{1}{|c|}{ Descrição } \\
\hline Luvissolo & 730,25 & 50,36 & $\begin{array}{l}\text { São de material mineral, } \\
\text { apresentando horizonte B } \\
\text { textural com argila de } \\
\text { atividade alta e saturação } \\
\text { por bases alta ma maior parte } \\
\text { dos primeiros 100 cm do } \\
\text { horizonte B. }\end{array}$ \\
\hline $\begin{array}{c}\text { Neossolo } \\
\text { litólico }\end{array}$ & 335,87 & 23,16 & $\begin{array}{l}\text { São solos rasos em que a } \\
\text { soma dos horizontes sobre a } \\
\text { rocha não ultrapassa 50 cm, } \\
\text { e são associados } \\
\text { normalmente a relevos mais } \\
\text { declivosos. }\end{array}$ \\
\hline Planossolo & 282,15 & 19,46 & $\begin{array}{l}\text { Ocorrem em áreas de cotas } \\
\text { baixas, planas a suave } \\
\text { onduladas e são geralmente } \\
\text { pouco profundos, com } \\
\text { horizonte superficial de } \\
\text { cores claras e textura } \\
\text { arenosa ou média, seguido } \\
\text { de um horizonte B plânico, } \\
\text { de textura média, argilosa } \\
\text { ou muito argilosa. }\end{array}$ \\
\hline 101,76 & 7,02 & $\begin{array}{l}\text { São solos minerais com } \\
\text { horizonte diagnóstico } \\
\text { vértico com pequena }\end{array}$ \\
\hline
\end{tabular}

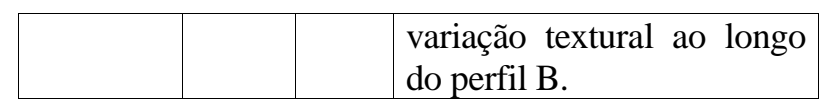

Fonte: EMBRAPA (2006).

(c) umidade do ar e (d) velocidade do vento. Esses dados foram obtidos no Instituto de Meteorologia (INMET), para o posto São Gonçalo, localizado nas coordenadas $6^{\circ} 45^{\prime} 00^{\prime \prime} \mathrm{S}$ de latitude e $38^{\circ} 12^{\prime} 36^{\prime \prime}$ $\mathrm{W}$ de longitude.

Quadro 2. Características dos postos pluviométricos $(\mathrm{P})$, estação meteorológica $(\mathrm{M}) \mathrm{e}$ posto fluviométrico $(\mathrm{F})$

\begin{tabular}{|c|c|c|c|c|c|}
\hline posto fluviométrico (F) & Posto & Tipo & $\begin{array}{c}\text { Lat. } \\
\left({ }^{\circ}\right)\end{array}$ & $\begin{array}{c}\text { Long. } \\
\left({ }^{\circ}\right)\end{array}$ & $\begin{array}{c}\text { Alt. } \\
(\mathbf{m})\end{array}$ \\
\hline 638059 & $\begin{array}{c}\text { Açude Lagoa } \\
\text { do Arroz }\end{array}$ & $\mathrm{P}$ & $-6,82$ & $-38,6$ & 350 \\
\hline 638046 & Açude Pilões & $\mathrm{P}$ & $-6,67$ & $-38,52$ & 255 \\
\hline 638032 & $\begin{array}{c}\text { Antenor } \\
\text { Navarro }\end{array}$ & $\mathrm{P}$ & $-6,74$ & $-38,45$ & 240 \\
\hline 638030 & Balanços & $\mathrm{P}$ & $-6,98$ & $-38,73$ & 400 \\
\hline 638029 & Barra do Juá & $\mathrm{P}$ & $-6,53$ & $-38,57$ & 500 \\
\hline 638108 & $\begin{array}{c}\text { Cachoeira dos } \\
\text { Índios }\end{array}$ & $\mathrm{P}$ & $-6,93$ & $-38,68$ & 325 \\
\hline 638115 & Poço Dantas & $\mathrm{P}$ & $-6,4$ & $-38,5$ & 295 \\
\hline 638105 & Santa Helena & $\mathrm{P}$ & $-6,73$ & $-38,64$ & 200 \\
\hline 638035 & Uiraúna & $\mathrm{P}$ & $-6,52$ & $-38,42$ & 300 \\
\hline 82689 & São Gonçalo & $\mathrm{M}$ & $-6,75$ & $-38,21$ & 233 \\
\hline 37260000 & $\begin{array}{c}\text { Antenor } \\
\text { Navarro }\end{array}$ & $\mathrm{F}$ & $-6,74$ & $-38,45$ & - \\
\hline
\end{tabular}

Fonte: ANA (2017). 
Revista Brasileira de Geografia Física v11, n.03 (2018) 1137-1150.
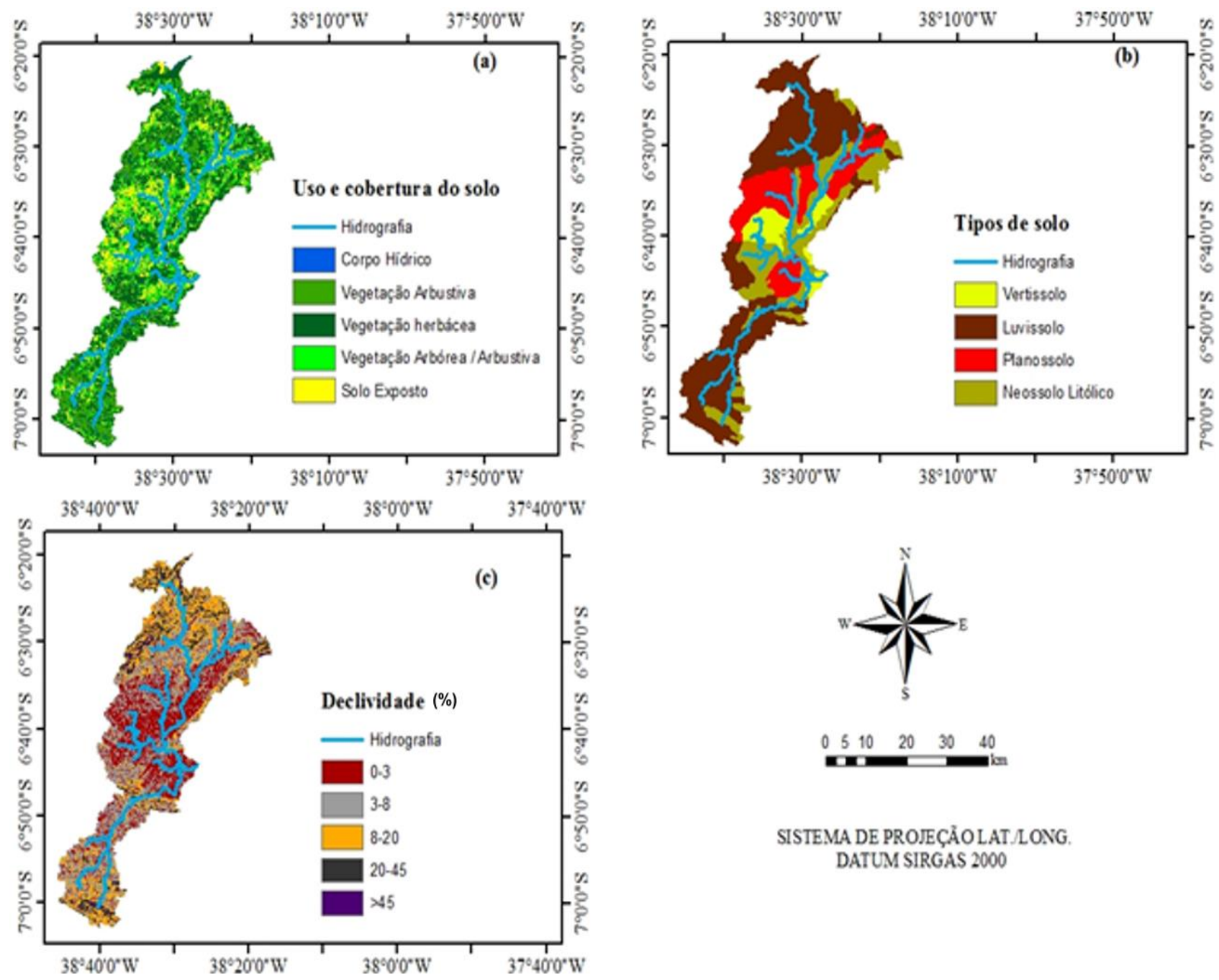

SISTEMA DE PROJEÇÃO LAT/LONG

DATUM SIRGAS 2000

Figura 2. Mapas da bacia do Alto Rio do Peixe: (a) cobertura do solo, (b) tipos de solo e (c) declividade

Fonte: (a) INPE (2016), (b) IBGE (2015) e (c) INPE (2016).

As declividades foram obtidas a partir do modelo digital de elevação com resolução de $30 \mathrm{~m}$, disponível no site do Instituto Nacional de Pesquisas Espaciais (INPE). As classes de declividade da bacia hidrográfica foram definidas de acordo com a EMBRAPA (1979).

\section{O modelo SWAT}

O modelo SWAT (Arnold e Allen, 1996) é um modelo distribuído e de base semi-física, desenvolvido para simular vazão e erosão e os impactos decorrentes das alterações no uso do solo em bacias hidrográficas. Esse modelo foi desenvolvido na década de 1990, nos Estados Unidos, pelo Agricultural Research Service e pela Texas A \& M University. O SWAT é um modelo hidrossedimentológico que permite a simulação de diferentes processos físicos em escala de bacia hidrográfica.

O modelo SWAT baseia-se em um conjunto de dados obtidos no campo como precipitação, vazão, evapotranspiração, irradiação solar, entre tantos outros, que associados são trabalhados de forma integrada em um SIG (Silva, 2010). Esta ferramenta está sendo cada vez mais utilizada em nível mundial para as mais diversas linhas de pesquisas por se tratar de um modelo semi-distribuído, capaz de espacializar os processos físicos em escala de bacia hidrográfica, e por levar em consideração informações sobre clima, hidrologia, propriedades do solo, uso e ocupação do solo, topografia, entre outros parâmetros que possibilitam a obtenção de resultados mais robustos sobre processos físicos muitas vezes complexos (Gassman et al., 2007).

Segundo Neitsch et al. (2011), a hidrologia do modelo SWAT está baseada na seguinte equação:

$\mathrm{SW}_{\mathrm{t}}=\mathrm{W}_{0}+\sum_{\mathrm{t}=1}^{\mathrm{t}}\left(\mathrm{R}_{\mathrm{i}}-\mathrm{Q}_{\mathrm{i}}-\mathrm{ET}_{\mathrm{i}}-\mathrm{P}_{\mathrm{i}}-\mathrm{QR}_{\mathrm{i}}\right)$

sendo $\mathrm{SW}_{\mathrm{t}}$ a quantidade em $\mathrm{mm}$ da água encontrada no solo no fim do dia, enquanto $\mathrm{W}_{\mathrm{o}}$ é a

Nascimento, J. M., Frade, T. G., Silva, R. M. 
quantidade no início do dia. Os parâmetros $R_{i}, Q_{i}$, $\mathrm{ET}_{\mathrm{i}}, \mathrm{P}_{\mathrm{i}}$ e $\mathrm{QR}_{\mathrm{i}}$ são a precipitação, o escoamento superficial, quantidade de evapotranspiração, quantidade de água presente na zona vadosa e a quantidade de água em fluxo de retorno, respectivamente. Todos medidos em mm por dia.

O escoamento superficial é determinado por:

$$
\mathrm{Q}_{\mathrm{i}}=\frac{\left(\mathrm{R}_{\mathrm{i}}-0,2 \mathrm{~S}\right)^{2}}{\left(\mathrm{R}_{\mathrm{i}}+0,8 \mathrm{~S}\right)}
$$

sendo S o parâmetro de retenção de água no solo, calculado da seguinte forma:

$$
\mathrm{S}=25,4\left(\frac{100}{\mathrm{CN}}-10\right)
$$

em que $\mathrm{CN}$ consiste no grau de permeabilidade do solo.

Calibração, validação e índices de desempenho

Após a simulação do escoamento foi realizada a calibração em escala de tempo mensal para o período compreendido entre os anos de 2005 a 2010, sendo que os anos de 2002 a 2004 foram utilizados para o aquecimento do modelo, como apresentado no estudo de Silva et al. (2016). Optou-se por utilizar o período de aquecimento do modelo em virtude desse procedimento auxiliar o melhor ajuste das condições climáticas iniciais pelo modelo, como umidade inicial, crescimento da vegetação e balanço climatológico da bacia, o que permite a melhor parametrização do modelo.

A calibração dos parâmetros foi realizada utilizando o software de autocalibração, chamado SWAT-CUP (Abbaspour et al., 2007), a partir do método SUFI-2. Para a calibração do modelo foram utilizados 19 parâmetros, visto que esses parâmetros influenciam diretamente no escoamento para as condições das bacias existentes na região Nordeste do Brasil, como mostrado nos estudos de Aragão et al. (2013), Dantas et al. (2015) e Santos (2015).

Na calibração automática, os valores dos parâmetros são modificados a cada iteração, buscando o melhor ajuste entre os escoamentos observados e estimados.

Para a calibração dos parâmetros do modelo foram realizadas 500 iterações, sendo que a cada iteração, os valores de cada parâmetro são modificados simultaneamente, o que agiliza o processo de calibração. Ao final da calibração, o SWAT-CUP calcula índices estatísticos que analisam o desempenho dos resultados obtidos, mostrando: (a) os valores de cada parâmetro, (b) os valores calculados para os índices de desempenho do modelo, (c) os valores simulados para a variável indicada (nesse caso a vazão) e (d) a iteração que apresentou o melhor ajuste.

Para a análise da comparação entre as vazões observadas e as simuladas foram utilizados os métodos estatísticos: (a) coeficiente de correlação $\left(R^{2}\right)$ e (b) coeficiente de eficiência de Nash-Sutcliffe (1970). O R ${ }^{2}$ é calculado por:

$$
R^{2}=\left(\frac{\sum_{i=1}^{n}\left(y_{i}-y_{m}\right)\left(x_{i}-x_{m}\right)}{\sqrt{\sum_{i=1}^{n}\left(y_{i}-y_{m}\right)\left(x_{i}-x_{m}\right)^{2}}}\right)^{2}
$$

sendo $\mathrm{x}_{\mathrm{i}}$ os valores observados; $\mathrm{x}_{\mathrm{m}}$ a média dos valores observados; $y_{i}$ os valores calculados pelo modelo; $\mathrm{y}_{\mathrm{m}}$ a média dos valores calculado pelo modelo; e $\mathrm{n}$ o número de eventos. $\mathrm{O} \mathrm{R}^{2}$ não deve ser considerado sozinho, mas, sempre aliado a outros diagnósticos do modelo. Por isso, também foi verificado o coeficiente de eficiência NashSutcliffe (E) calculado pela fórmula:

$$
\mathrm{NS}=1-\left(\sum_{\mathrm{i}=1}^{\mathrm{n}}\left(\mathrm{E}_{\mathrm{m}}-\mathrm{E}_{\mathrm{s}}\right)^{2} / \sum_{\mathrm{i}=1}^{\mathrm{n}}\left(\mathrm{E}_{\mathrm{m}}-\overline{\mathrm{E}_{\mathrm{s}}}\right)^{2}\right)
$$

sendo $E_{m} o$ evento observado; $E_{s} o$ evento simulado pelo modelo; $\overline{\mathrm{E}_{\mathrm{s}}}$ a média do evento observado no período de simulação; e $n$ o número de eventos. $\mathrm{O}$ E analisa o comportamento dos dados simulados em relação aos dados observados, podendo variar de $-\infty$ (infinito negativo) a 1 , no qual NS $=1$ indica um ajuste perfeito.

Em seguida foi realizada uma análise de sensibilidade dos parâmetros utilizados na calibração. Esta análise procurou identificar quais os parâmetros que apresentaram maior sensibilidade na bacia. Após a etapa da calibração foi realizada a validação do modelo utilizando dados mensais de vazão para o período de janeiro de 2011 a dezembro de 2014. A validação é a última etapa de verificação de como se comportou o modelo hidrológico. Usa-se um período de dados diferentes da calibração, simulando o escoamento superficial com os parâmetros determinados na calibração (Arnold e al., 2012).

\section{Resultados e discussão}

A Figura 3 mostra o comportamento da análise de sensibilidade dos 19 parâmetros calibrados para a bacia. $\mathrm{O}$ índice $t$-stat indica o grau 
Revista Brasileira de Geografia Física v11, n.03 (2018) 1137-1150.

da sensibilidade, e quanto maior for o seu valor, mais sensível é o parâmetro. Já o $p$-value determina a significância da sensibilidade dos parâmetros, sendo que valores próximos de zero apresentam maior significância na modelagem específica (Abbaspour et al., 2015).

O Quadro 3 mostra os parâmetros considerados mais sensíveis, os processos hidrológicos aos quais estão relacionados e suas descrições dentro do modelo SWAT. Desta forma, para a calibração da vazão da bacia do Alto Rio do Peixe, foram considerados como mais sensíveis os 10 parâmetros que apresentaram um p-value inferior a 0,4 .

Além de caracterizar os parâmetros mais sensíveis e passíveis de calibração, a análise de sensibilidade também é imprescindível para verificar quanto o modelo conseguiu estimar os processos hidrológicos predominantes na bacia.

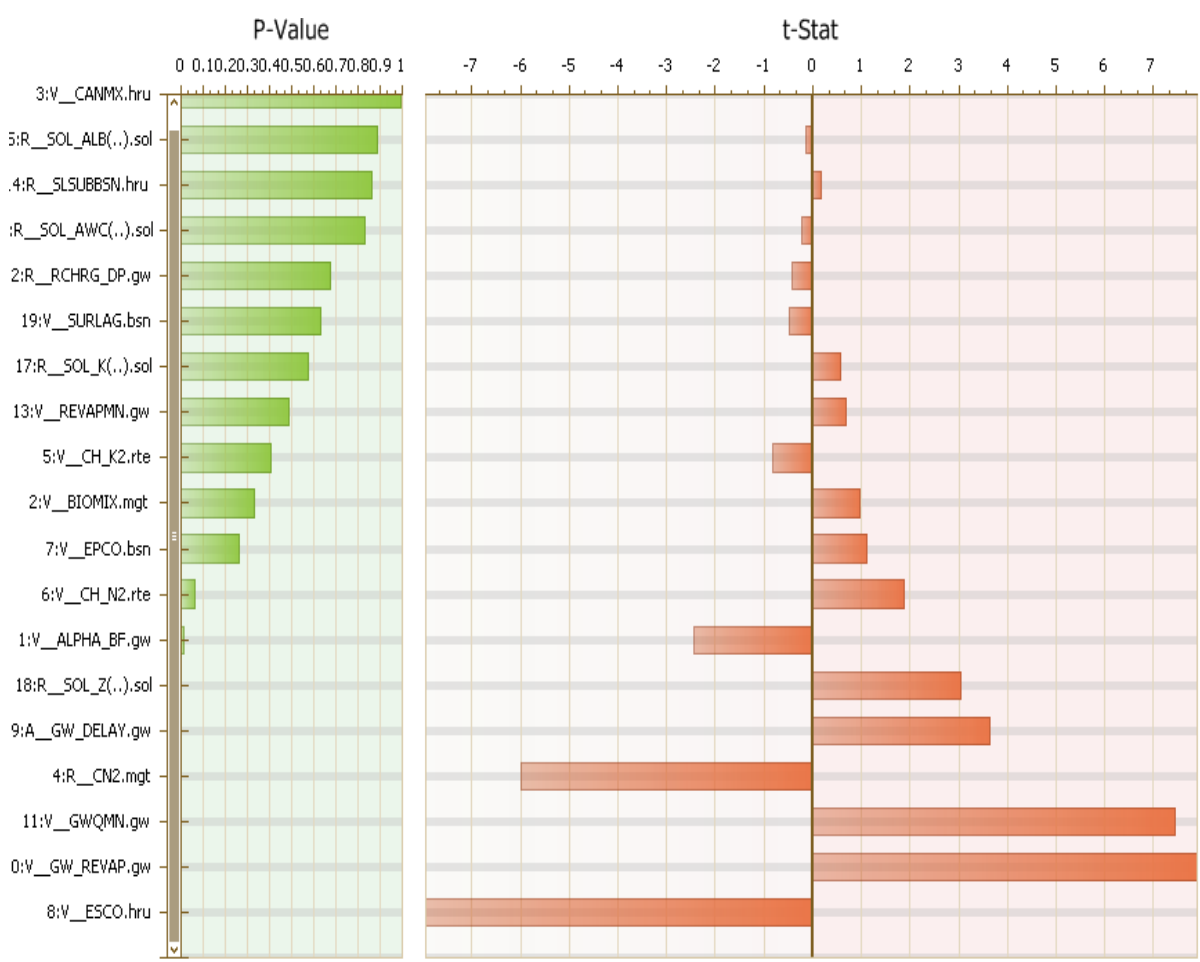

Figura 3. Análise de sensibilidade dos 19 parâmetros utilizados neste estudo.

Fonte: Os Autores.

Quadro 3. Parâmetros mais sensíveis para a bacia do Alto Rio do Peixe.

\begin{tabular}{|l|c|c|}
\hline \multicolumn{1}{|c|}{ Parâmetros } & $\begin{array}{c}\text { Código do } \\
\text { Parâmetro }\end{array}$ & Processos \\
\hline Coeficiente de compensação da evaporação de água no solo & Esco & Evapotranspiração \\
\hline $\begin{array}{l}\text { Coeficiente de controle do fluxo da água entre as zonas } \\
\text { saturada e não saturada }\end{array}$ & Gw_Revap & Escoamento de base \\
\hline $\begin{array}{l}\text { Profundidade da água subterrânea necessária para ocorrer fluxo } \\
\text { de retorno }\end{array}$ & Gwqmn & Escoamento de base \\
\hline Valor da curva número para a condição de solo úmido & Cn2 & Escoamento superficial \\
\hline Tempo de recarga do aquífero & Gw_Delay & Escoamento de base \\
\hline Profundidade da camada de solo & Sol_Z & $\begin{array}{c}\text { Escoamento superficial/ } \\
\text { Escoamento de base/ } \\
\text { Evapotranspiração/ } \\
\text { Armazenamento }\end{array}$ \\
\hline Fator de recessão de escoamento de base & Alpha_Bf & Escoamento de base \\
\hline Coeficiente de Manning do canal & Ch_N2 & Escoamento superficial \\
\hline Coeficiente de compensação da absorção de água pelas plantas & Epco & Infiltração \\
\hline Eficiência da mistura biológica do solo & Biomix & Infiltração \\
\hline
\end{tabular}


Revista Brasileira de Geografia Física v11, n.03 (2018) 1137-1150.

Outro aspecto a ser destacado é que essa análise é extremamente dependente da estatística de precisão utilizada para quantificar a sensibilidade, ou seja, o coeficiente E sofrerá mais influência de parâmetros vinculados ao escoamento direto. Deve-se ressaltar que os parâmetros mais sensíveis, apresentados na Quadro 3, estão de acordo com a predominância dos processos hidrológicos na bacia do Alto Rio do
Peixe, com predominância de escoamento de base, seguido do escoamento superficial direto.

O Quadro 4 mostra os valores otimizados dos parâmetros utilizados na calibração do modelo SWAT para a bacia do Alto Rio do Peixe. Percebese que a parametrização do modelo representou satisfatoriamente os aspectos físicos do solo e da vegetação da bacia.

Quadro 4. Valores otimizados dos parâmetros utilizados na calibração do modelo SWAT

\begin{tabular}{|c|c|c|}
\hline Parâmetros & Fator de recessão de escoamento de base (dias) & Valores \\
\hline Alpha_Bf & Eficiência da mistura biológica do solo (adimensional) & 0,105 \\
\hline Biomix & Quantidade máxima de água interceptada pela vegetação (mm) & 0,537 \\
\hline Canmx & Valor da curva número para a condição de solo úmido (adimensional) & 2,591 \\
\hline Cn2 & Condutividade hidráulica efetiva do canal (mm/h) & $-0,0662$ \\
\hline Ch_K2 & Coeficiente de Manning do canal (adimensional) & 3,635 \\
\hline Ch_N2 & Coeficiente de compensação da absorção de água pelas plantas (adimensional) & 0,1059 \\
\hline Epco & Tempo de recarga do aquífero (dias) & 0,683 \\
\hline Esco & Coeficiente de compensação da evaporac̃ão de água no solo (adimensional) & 0,7365 \\
\hline Gw_delay & (adimensional) & $-18,75$ \\
\hline Gw_revap & Coeficiente de controle do fluxo da água entre as zonas saturada e não saturada & \multirow{2}{*}{0,1487} \\
\hline Gw_qmn & Profundidade da água subterrânea necessária para ocorrer fluxo de retorno (mm) & 659 \\
\hline Rchrg_Dp & Fração de água percolada para o aquífero profundo (fração) & $-0,00643$ \\
\hline Revapmn & Limite de água no solo para que ocorra ascensão capilar na zona saturada (mm) & 1,91 \\
\hline Slsubbsn & Comprimento da declividade média (m) & $-0,1095$ \\
\hline Sol_Alb & Albedo do solo (adimensional) & 0,0735 \\
\hline Sol_Awc & Capacidade de armazenamento de água no solo (mm/mm) & $-0,1615$ \\
\hline Sol_K & Condutividade hidráulica saturada do solo $(\mathrm{mm} / \mathrm{h})$ & $-0,0755$ \\
\hline Sol_Z & Profundidade da camada de solo (mm) & 0,1185 \\
\hline Surlag & Tempo de retardo de escoamento superficial (dias) & 8,664001 \\
\hline
\end{tabular}
Fonte: Os Autores (2018).

A bacia do Alto Rio do Peixe foi dividida em 85 sub-bacias para modelar a resposta hidrológica do escoamento. Os resultados da simulação para o período de 2002 a 2010 mostraram que o modelo SWAT representou satisfatoriamente a vazão do Alto Rio do Peixe no período estudado, com vazão média observada de $4,7 \mathrm{~m}^{3} / \mathrm{s}$ e vazão média simulada de $5,8 \mathrm{~m}^{3} / \mathrm{s}$, como mostra a Figura 4. 


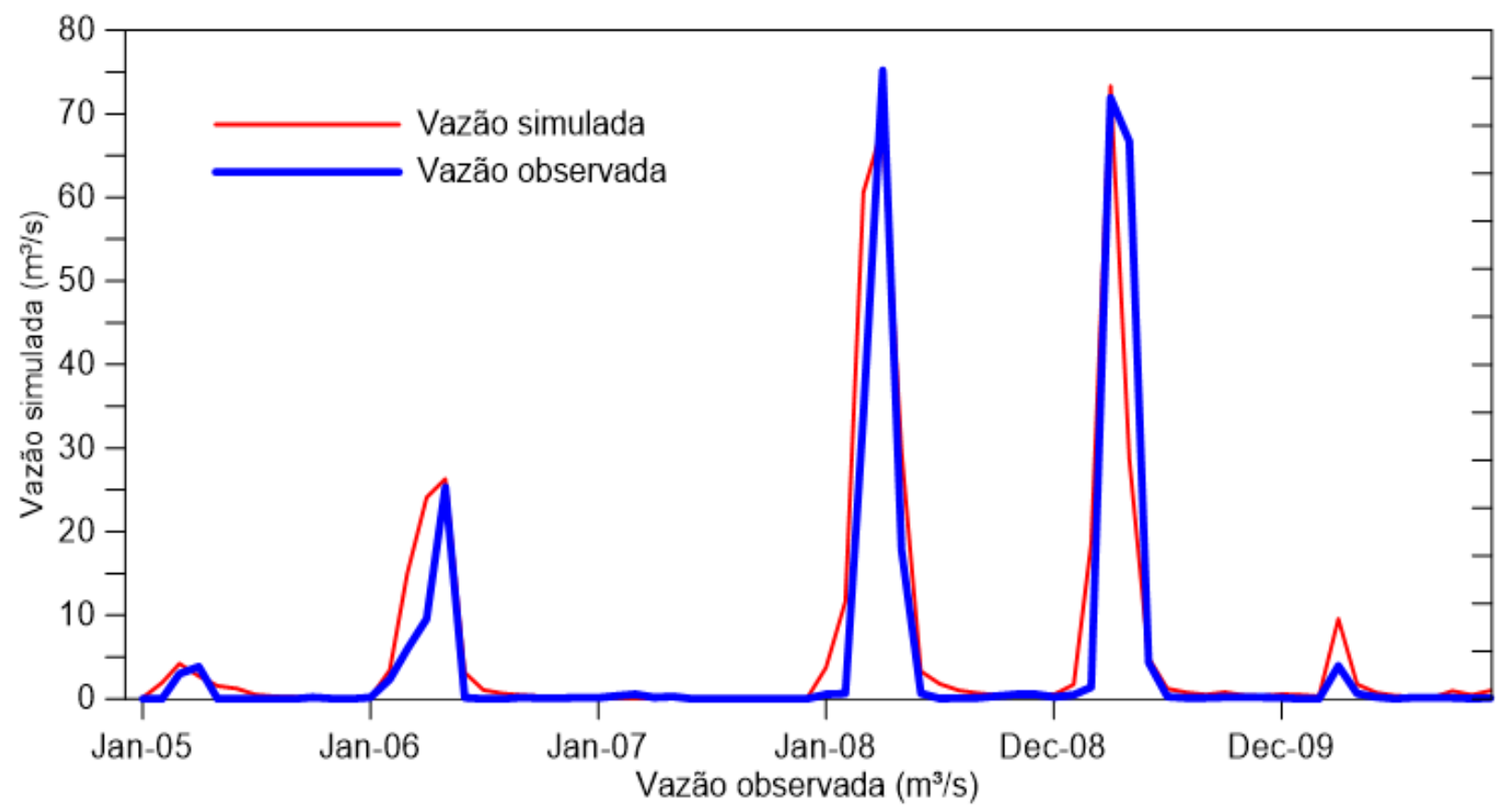

Figura 4. Comparação entre os dados observados e os simulados no período de calibração de 2005 2010 para o posto fluviométrico Antenor Navarro.

Os resultados obtidos no período de calibração mostraram que o modelo SWAT superestimou os dados observados e poucos eventos extremos na bacia e mostra também que o modelo conseguiu acompanhar a ascensão e o recesso do hidrograma. O modelo conseguiu ajustar muito bem todos os picos de vazão no período de calibração, bem como as pequenas vazões. Entretanto, no período da validação, o modelo não conseguiu ajustar satisfatoriamente as grandes vazões nos primeiros meses de 2011 e superestimou a vazão nos meses de janeiro, fevereiro e março no ano de 2012, mas conseguiu representar bem a vazão estimada quando comparada com a vazão observada.

Os índices de desempenho da calibração entre os valores observados e simulados foram de:
$\mathrm{NS}=0,81$ e $\mathrm{R}^{2}=0,82$. Segundo Moriasi et al. (2007), esses valores são considerados "muito bons". O que significa uma boa parametrização do modelo em superestimar o escoamento superficial médio.

No que tange a validação do modelo para o período de 2011 a 2014 também foi obtido um bom resultado, com vazão média observada de $1 \mathrm{~m}^{3} / \mathrm{s}$ e simulada de $1,3 \mathrm{~m}^{3} / \mathrm{s}$, como mostra a Figura 5 . No período de validação percebeu-se uma diminuição nas taxas de escoamento devido a fatores naturais (climáticos) ou antrópicos, o que pode estar relacionada tanto a causas antrópicas, como fragmentação do uso e ocupação do solo e construção de reservatórios de pequeno e médio porte, quanto a alta variabilidade hidrográfica da bacia (Silva et al., 2018; Silva, 2015). 


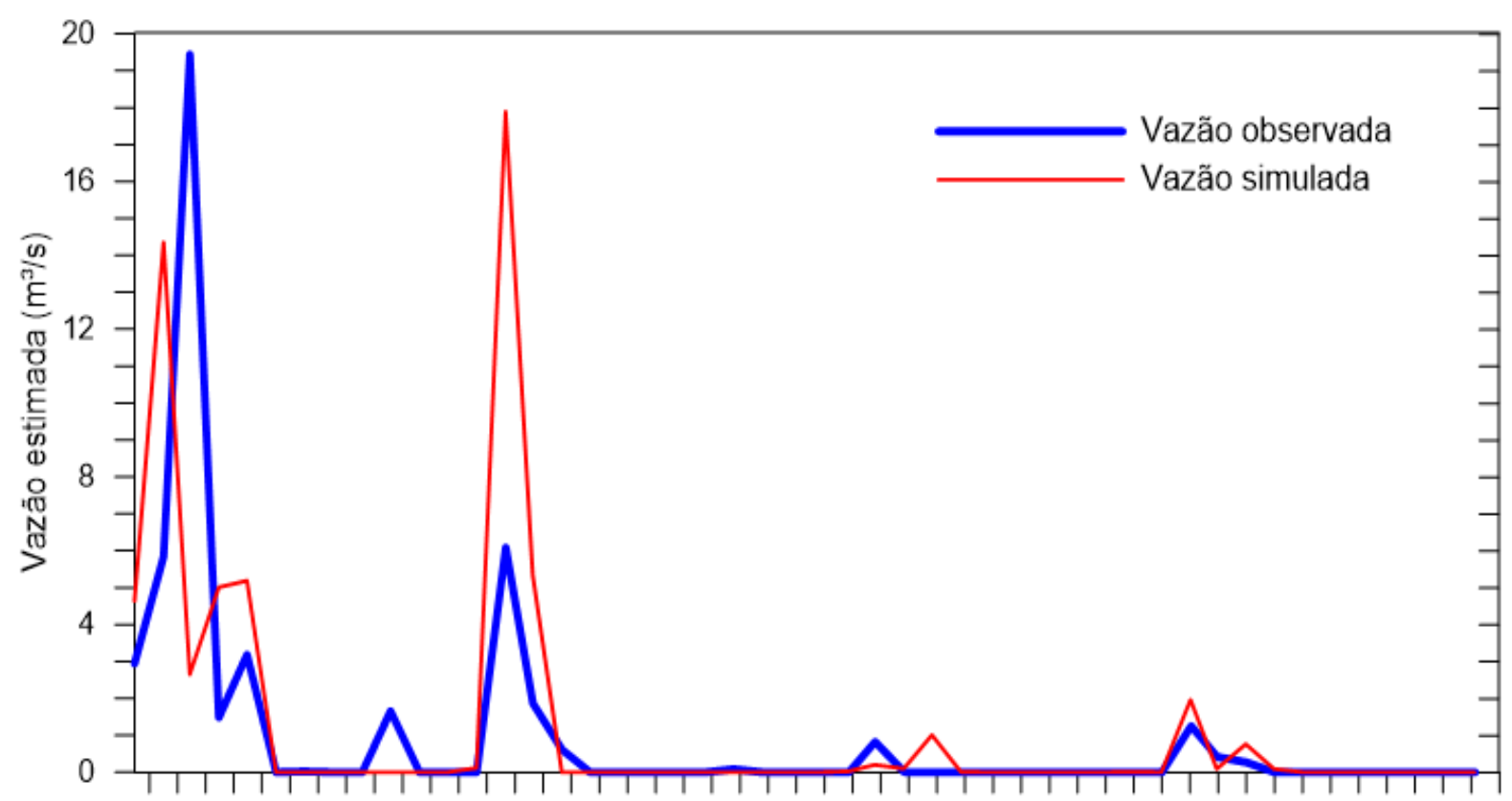

Jan-11 May-11 Sep-11 Jan-12 May-12 Sep-12 Jan-13 May-13 Sep-13 Jan-14 May-14 Aug-14 Dec-14

\section{Vazão observada $\left(\mathrm{m}^{3} / \mathrm{s}\right)$}

Figura 5. Comparação entre os dados observados e simulados no período de validação de 2011-2014 para o posto fluviométrico Antenor Navarro.

Em termos gerais, a calibração e a validação para vazão simulada para o posto Antenor Navarro foram satisfatórias, com valores de NS e $\mathrm{R}^{2}$ considerados bons.

Pinheiro e Morais (2010) ao estudarem a influência de barramentos de pequeno e médio porte na bacia hidrográfica do Rio Catú, no Estado do Ceará, verificaram que a construção do reservatório Catú-Cizento alterou a contribuição do volume de água da bacia para o estuário do rio, pois antes da construção do reservatório o volume registrado na Lagoa do Catú (jusante) foi de $5 \times 10^{7}$ $\mathrm{m}^{3}$, e após a construção passou a ser de $4 \times 10^{6} \mathrm{~m}^{3}$.

As Figuras $6 \mathrm{a}-\mathrm{b}$ mostram as relações entre os valores dos escoamentos observados e estimados nos períodos de calibração e validação para a bacia do Alto Rio do Peixe. Os resultados da análise de dispersão entre os escoamentos observados e calculados não permitem alegar a ocorrência de uma tendência ou erro sistemático do modelo SWAT em superestimar ou subestimar as vazões observadas, seja nas pequenas ou nos picos de vazões, para ambos os períodos testados, sobretudo no período de validação, quando o modelo não conseguiu ajustar satisfatoriamente os maiores valores de escoamentos observados.

Apesar desse comportamento, os índices NS e $\mathrm{R}^{2}$ podem ser considerados bons (Moriasi et al., 2007), e refletem os hidrogramas apresentados. Esse comportamento dos hidrogramas pode ser atribuído à influência, sobretudo, aos parâmetros
$\mathrm{Cn} 2$, que se refere ao uso e à cobertura do solo e ao Sol_Z, que influencia nos escoamentos superficial e de base, pois controla o armazenamento de água no solo.

As Figuras $7 \mathrm{a}-\mathrm{b}$ mostram as curvas de permanência das vazões mensais observadas e 
Revista Brasileira de Geografia Física v11, n.03 (2018) 1137-1150.

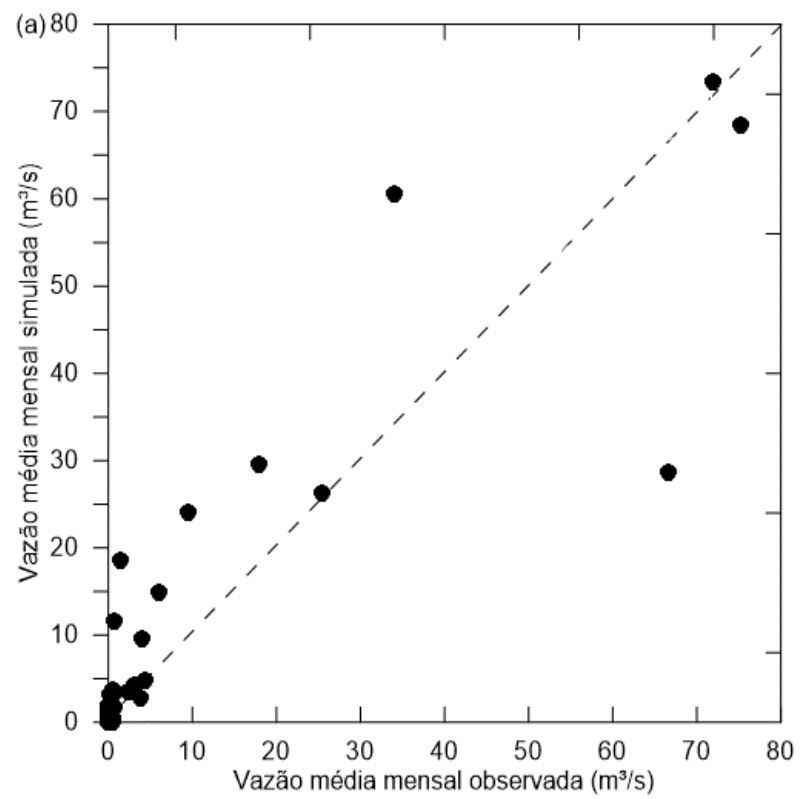

permanência $4 \%$ até $95 \%$, o modelo
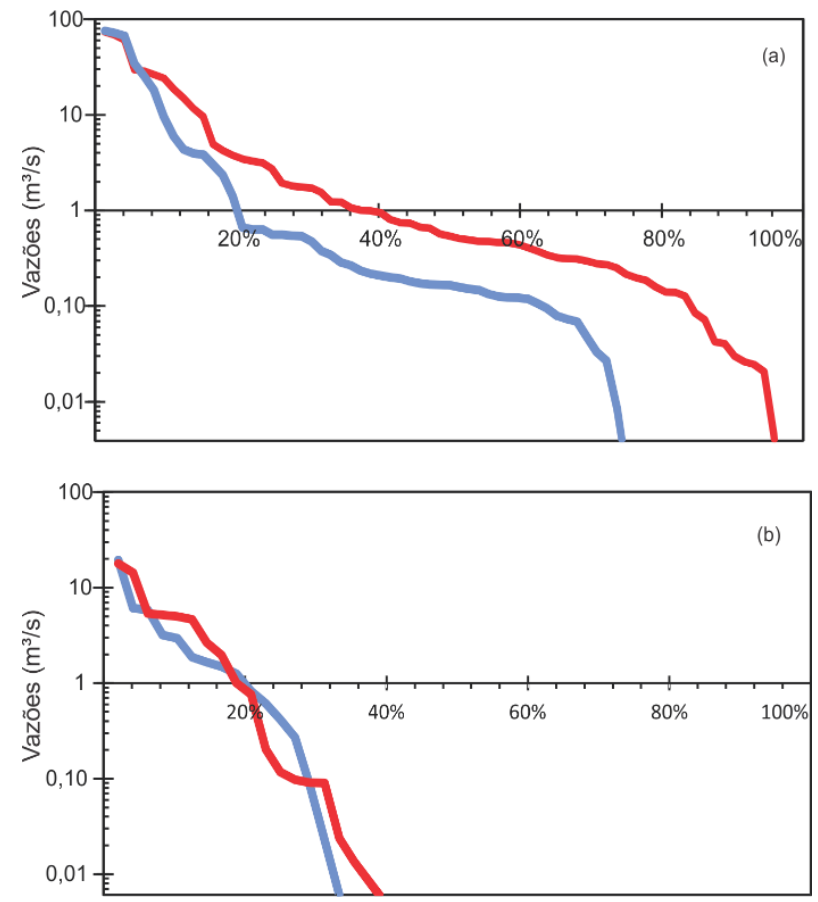

Figura 7. Curvas de permanência das vazões mensais observadas e calculadas para a bacia do alto rio do Peixe: (a) no período de calibração e (b) no período de validação.

Fonte: Os Autores (2018).

SWAT apresentou maiores valores do que os observados, mas seguindo uma curva de permanência relativamente paralela à observada. Para as maiores vazões, com permanência inferior a 5\%, houve maior correspondência entre as curvas de permanência entre os valores observados e calculados pelo modelo. A despeito das dificuldades encontradas na comparação visual entre os hidrogramas observados e calculados, os valores dos índices de desempenho do modelo são no mesmo patamar das obtidas na maioria das demais aplicações do modelo para bacias de rios intermitentes. Por exemplo, o valor de E e $\mathrm{R}^{2}$ obtidos foram na mesma faixa ou até superior aos alcançados por Silva et al. (2016), Felix e Paz (2016), Medeiros e Silva (2014).

\section{Conclusão}

Este trabalho apresentou a aplicação de um modelo SWAT para a bacia do Alto Rio do Peixe, localizada no Estado da Paraíba. Diante do que foi apresentado, o modelo SWAT mostrou um bom desempenho para o período de calibração da bacia do Alto Rio do Peixe. Os valores obtidos para os índices de Eficiência de Nash-Sutcliffe (E) e $\mathbf{R}^{2}$ foram considerados muito bons para o período de

Nascimento, J. M., Frade, T. G., Silva, R. M. 
calibração, mas foi inferior no período de validação. Com base nos resultados obtidos $o$ modelo SWAT conseguiu reproduzir consistentemente os escoamentos mensais estimados e o volume escoado nos períodos.

A análise de sensibilidade mostrou que os parâmetros mais sensíveis foram GW_REVAP, GWQMN, CN2, GW_DELAY e EPCO, que estão relacionados à condição de umidade do solo e ao balanço hídrico e ESCO, SOL_Z, ALPHA_BF, BIOMIX e CH_N2, que estão associados à infiltração da água e à profundidade do solo.

Desta forma, os resultados obtidos são animadores, e dessa forma, o modelo pode ser considerado como uma ferramenta promissora na simulação de vazões em qualquer ponto da bacia.

\section{Agradecimentos}

Os autores agradecem ao CNPq pelo auxílio financeiro proporcionado pelo projeto "Mudanças climáticas e no uso do solo e seus impactos nos recursos hídricos na bacia do Açude Epitácio Pessoa, Paraíba" (Processo Nº. 408631/2016-3), e pelas bolsas de Iniciação Científica dos dois primeiros autores e de produtividade em pesquisa do terceiro autor (Processo No 304540/2017-0).

\section{Referências}

Abbaspour, K.C., Rouholahnejad, E., Vaghefi, S., Srinivasan, R., Yang, H., Kløve, B., 2015. A continental-scale hydrology and water quality model for Europe: calibration and uncertainty of a high-resolution large-scale SWAT model. Journal of Hydrology 524, 733-752.

Abbaspour, K.C., Yang, J., Maximov, I., Siber, R., Bogner, K., Mieleitner, J., Zobrist, J., Srinivasan, R., 2007. Modelling hydrology and water quality in the pre-alpine/alpine Thur watershed using SWAT. Journal of Hydrology 333, 413-430.

ANA. Agência Nacional de Águas, 2015. Sistema Nacional de Informações sobre Recursos Hídricos. Disponível: http://www.snirh.gov.br. Acesso: 19 set. 2017.

Andrade, M.A., Mello, C.R. Beskow, S., 2013. Simulação hidrológica em uma bacia hidrográfica representativa dos Latossolos na região Alto Rio Grande, MG. Revista Brasileira de Engenharia Agrícola e Ambiental 17, 69-76.

Aragão, R., Cruz, M.A.S., Amorim, J.R.A., Mendonça, L.C., Figueiredo, E.E., Srinivasan, V.S., 2013. Análise de sensibilidade dos parâmetros do modelo SWAT e simulação dos processos hidrossedimentológicos em uma bacia no Agreste Nordestino. Revista Brasileira de Ciência do Solo 37, 1091-1102.

Araújo, S.M.S., 2011. A região semiárida do nordeste do Brasil: questões ambientais e possibilidades de uso sustentável dos recursos. Revista Científica da FASETE 5, 89-98.

Arnold, J.G., Allen, P.M., 1996. Estimating hydrologic budgets for three Illinois watersheds. Journal of Hydrology 176, 55-57.

Arnold, J.G., Moriasi, D.N., Gassman, P.W., Abbaspour, K.C., White, M.J., Srinivasan, R., 2012. SWAT: model use calibration and validation. Transactions of the ASABE 55, 1494-1508.

Brandão, M.H.M., 2005. Índice de degradação ambiental na bacia hidrográfica do Rio do Peixe - PB. Tese (Doutorado). Recife, UFPE.

BRASIL. Ministério da Agricultura. 1972, I Levantamento exploratório-reconhecimento de solos do estado da Paraíba. II - Interpretação para uso agrícola dos solos do Estado da Paraíba. Rio de Janeiro.

Bressiani, D.A., Gassman, P.W., Fernandes, J.G., Garbossa, L.H.P., Srinivasan, R., Bonumá, N.B., Mendiondo, E.M., 2015a. Review of Soil and Water Assessment Tool (SWAT) applications in Brazil: challenges and prospects. International Journal of Agricultural and Biological Engineering 8, 9-35.

Bressiani, D.A., Srinivasan, R., Jones, C.A., Mendiondo, E.M., 2015b. Effects of spatial and temporal weather data resolutions on streamflow modeling of a semi-arid basin, Northeast Brazil. International Journal of Agricultural and Biological Engineering 8, 125139.

Cabral, L.N., Xavier, J.M.V., Rocha, D.C.M 2013. Soluções para o problema da água no semiárido nordestino: as secas e suas consequências. Anais... I Workshop Internacional sobre água no semiárido Brasileiro. Campina Grande.

Cirilo, J.A., Góes, V.C., Asfora, M.C., 2007. Integração das águas superficiais e subterrâneas, in: Cirilo, J.A., Cabral, J.J.S.P., Ferreira, J.P.L., Oliveira, M.J.P.M., Leitão, T.E., Montenegro, S.M.G.L. \& Góes, V.C. (orgs.), O uso sustentável dos recursos hídricos em regiões semi-áridas. 1. ed. Editora da ABRH, Porto Alegre, pp. 167-175. 
Dantas, J.C., SILVA, M.A. Silva, R.M., Vianna, P.C.G., 2015. Simulação vazão-erosão usando o modelo SWAT para uma grande bacia da região semiárida da Paraíba. Geociências 34, 816-827.

EMBRAPA. Empresa Brasileira de Pesquisa Agropecuária, 1979. Serviço Nacional de Levantamento e Conservação de Solos. Súmula da X Reunião Técnica de Levantamento de Solos. Rio de Janeiro.

EMBRAPA. Empresa Brasileira de Pesquisa Agropecuária, 2006. Sistema Brasileiro de Classificação de Solos. 2. ed. EMBRAPA, Rio de Janeiro.

Felix, V.S., Paz, A.R., 2016. Representação dos processos hidrológicos em bacia hidrográfica do semiárido paraibano com modelagem hidrológica distribuída. Revista Brasileira de Recursos Hídricos 21, 556-569.

Gassman, P. W., Reyes, M. R., Green C. H., Arnold, J.G., 2007. The Soil and Water Assessment Tool: historical development, applications, and future research directions. Transaction of the ASABE 50, 1211-1250.

IBGE. Instituto Brasileiro de Geografia e Estatística, 2015. Mapas temáticos. Disponível: https://mapas.ibge.gov.br/tematicos.html. Acesso em: 15 de jun. 2015.

INPE. Instituto Nacional de Pesquisas Espaciais, 2015a. Banco de dados geomorfométricos do Brasil (TOPODATA). Disponível: http://www.dsr.inpe.br/topodata. Acesso: 15 jun. 2015.

Lima, A., Silva, D., Sampaio, J., 2011. As Tecnologias sociais como estratégia de convivência com a escassez de água no semiárido cearense. Revista Conexões - Ciência e Tecnologia 5, 9-21.

Marengo, J.A., 2006. Mudanças climáticas globais e seus efeitos sobre a biodiversidade: caracterização do clima atual e definição das alterações climáticas para o território brasileiro ao longo do século XXI. Brasília, MMA.

Medeiros, I.C., Silva, R.M., 2014. Análise da erosão hídrica na região semiárida da Paraíba usando o modelo SWAT acoplado a um SIG. Geociências 33, 457-471.

Moriasi, D.N., Arnold, J.G., Van Liew, M.W., Bingner, R.L., Harmel, R.D., Veith, T.L., 2007. Model evaluation guidelines for systematic quantification of accuracy in watershed simulations. Transactions of the ASABE 50, 885-900.

Nash, J.E., Sutcliffe, J.V., 1970. River flow forecasting through conceptual models I: a discussion of principles. Journal of Hydrology 10, 282-290.

Neitsch, S.L., Arnold, J.G., Kiniry, J.R., Williams, J.R., 2011. Soil and Water Assessment Tool: theoretical documentation - version 2009. Temple, Texas A\&M University.

ONU. Organização das Nações Unidas. 2015. Disponível: <https://nacoesunidas.org /ate-2030-planeta-pode-enfrentar-deficit-deagua-de-ate-40-alerta-relatorio-da-onu/> . Acesso: 20 dez. 2017.

Pinheiro, L.S., Morais, J.O., 2010. Interferências de barramentos no regime hidrológico do estuário do Rio Catú-Ceará-Nordeste do Brasil. Sociedade \& Natureza 22, 237-250.

Pinto, M.A.V.S., 1998. Diagnóstico ambiental da bacia do Rio do Peixe. Projetos de Execução Descentralizada. Sousa, MMA/PNMA/PED.

Rennó, C.D., Soares, J.V., 2008. Conceitos básicos de modelagem hidrológica. Capítulo 2. Disponível:

<http://www.dpi.inpe.br/cursos/tutoriais/model agem/cap2_modelos_hidrologicos.pdf> Acesso: 20 dez. 2017.

Santos, C.A.G., Brasil Neto, R.M., Passos, J.S.A., Silva, R.M., 2017. Drought assessment using a TRMM-derived standardized precipitation index for the Upper São Francisco River basin, Brazil. Environmental Monitoring and Assessment 189, 250-278.

Santos, J.Y.G., 2015. Análise espaço-temporal de processos hidrossedimentológicos na bacia do Rio Tapacurá (Pernambuco, Brasil). Tese (Doutorado). Recife, UFPE.

Silva, M.A., 2015. Modelagem hidrológica e das perdas do solo: suas relações com as formas de relevo e uso do solo na bacia do rio Taperoá-PB. Dissertação (Mestrado). João Pessoa, UFPB.

Silva, O., 2003. A Escassez de água no semiárido brasileiro. Dissertação (Mestrado). Campina Grande, UFCG.

Silva, R.M., Dantas, J.C., Beltrão, J.A., Santos, C.A.G., 2018. Hydrological simulation in a tropical humid basin in the Cerrado biome using the SWAT model. Hydrology Research 49, 116. 
Revista Brasileira de Geografia Física v11, n.03 (2018) 1137-1150.

Silva, R.M., Santos, C.A.G., Silva, V.C.L., Medeiros, I.C., Moreira, M., Corte-Real, J., 2016. Efeitos de cenários de uso do solo na vazão e produção de sedimentos na bacia do rio Cobres, Portugal. Geociências 35, 816-827.

Silva, R.M., Santos, C.A.G., Santos, J.Y.G., 2017. Evaluation and modeling of runoff and sediment yield for different land covers under simulated rain in a semiarid region of Brazil.
International Journal of Sediment Research 33, 582-598.

Silva, V.A., 2010. A utilização de técnicas de geoprocessamento para modelagem de cenários de uso e perda de solo na bacia hidrográfica do Rio Colônia no litoral sul da Bahia (Brasil). Anais... VI Seminário Latino Americano de Geografia Física e II Seminário Ibero Americano de Geografia Física Universidade de Coimbra, 2010. 